\title{
Sixfold excitations in electrides
}

\author{
Simin Nie $\odot,{ }^{1,2}$ B. Andrei Bernevig,,$^{3, *}$ and Zhijun Wang $\odot^{1,4, \dagger}$ \\ ${ }^{1}$ Beijing National Laboratory for Condensed Matter Physics, and Institute of Physics, Chinese Academy of Sciences, Beijing 100190, China \\ ${ }^{2}$ Department of Materials Science and Engineering, Stanford University, Stanford, California 94305, USA \\ ${ }^{3}$ Department of Physics, Princeton University, Princeton, New Jersey 08544, USA \\ ${ }^{4}$ University of Chinese Academy of Sciences, Beijing 100049, China
}

(Received 24 September 2020; accepted 9 February 2021; published 22 March 2021)

\begin{abstract}
Due to the lack of full rotational symmetry in condensed matter physics, solids exhibit new excitations beyond Dirac and Weyl fermions, of which the sixfold excitations have attracted considerable interest owing to the presence of maximum degeneracy in bosonic systems. Here, we propose that a single linear dispersive sixfold excitation can be found in the electride $\mathrm{Li}_{12} \mathrm{Mg}_{3} \mathrm{Si}_{4}$ and its derivatives. The sixfold excitation is formed by the floating bands of elementary band representation $A @ 12 a$ originating from the excess electrons centered at the vacancies (i.e., the $12 a$ Wyckoff sites). There exists a unique topological bulk-surface-edge correspondence for the spinless sixfold excitation, resulting in trivial surface "Fermi arcs" but topological hinge arcs. All gapped $k_{z}$ slices belong to a two-dimensional higher-order topological insulating phase, which is protected by a combined symmetry $\mathcal{T} \widetilde{S}_{4 z}$ and characterized by a quantized fractional corner charge $Q_{\text {corner }}=\frac{3|e|}{4}$. Consequently, the hinge arcs are obtained in the hinge spectra of the $\widetilde{S}_{4 z}$-symmetric rod structure. The state with a single sixfold excitation, stabilized by both nonsymmorphic crystalline symmetries and time-reversal symmetry, is located at the phase boundary and can be driven into various topologically distinct phases by explicit breaking of symmetries, making these electrides promising platforms for the systematic studies of different topological phases.
\end{abstract}

DOI: 10.1103/PhysRevResearch.3.L012028

In high-energy physics, Poincaré symmetry puts strong constraints on the standard model, leading to only three different types of fermions [1], i.e., Dirac, Weyl, and Majorana fermions. Although several kinds of particles (such as protons) are confirmed to be Dirac fermions, the signature of Weyl and Majorana fermions is still lacking in particle physics experiments. In contrast to the stagnant situation in highenergy physics, great progress has been made recently in the realization of their low-energy quasiparticles in condensed matter [2-20].

More interestingly, due to the less constraints placed by the space group (SG) symmetries, condensed matter systems can host various new types of quasiparticles without counterparts in high-energy physics [21], such as threefold spin-1 excitations [21-24], fourfold spin-3/2 Rarita-Schwinger-Weyl (RSW) fermions [25], sixfold excitations [21], etc. Many exotic properties have been proposed in these semimetals, such as "Fermi-arc" surface states, anomalous negative magnetoresistance [21], and hinge arc states [26]. Similar to the fourfold Dirac fermions made up of two Weyl fermions with opposite chirality [Figs. 1(a) and 1(b)], the sixfold excitation can be

\footnotetext{
*bernevig@princeton.edu

†wzj@iphy.ac.cn
}

Published by the American Physical Society under the terms of the Creative Commons Attribution 4.0 International license. Further distribution of this work must maintain attribution to the author(s) and the published article's title, journal citation, and DOI. considered as two threefold spin-1 excitations with opposite chirality [Figs. 1(c) and 1(d)]. Although the spin-1 excitations and spin-3/2 RSW fermions have been proposed in CoSi [22] and verified in experiments [27,28], the sixfold excitations in the spinless systems have not been well investigated in both experimental and theoretical considerations. Recently, the signature of spinful sixfold fermions [considering spinorbit coupling (SOC)] has been observed in $\mathrm{PdSb}_{2}$ [29-32], but the presence of many trivial Fermi surfaces renders the problem very complicated for a simple understanding. Unlike the spin-3/2 (or spin-1/2) fermions, the spin-1 excitations, and the twofold Dirac fermions in two-dimensional (2D) graphene, which cannot exist alone in the Brillouin zone (BZ), we show that a single linear dispersive sixfold excitation can be protected near the Fermi level $\left(E_{\mathrm{F}}\right)$ in three-dimensional (3D) crystals.

Recently, electrides have been found in inorganic crystals [33-37]. They are defined as ionic crystals with excess electrons serving as anions confined in particular cavities. Compared with the electrons bounded by nuclei in atomic insulators [in the literature they are classified as trivial insulators with valence bands (VBs) originating from real atomic orbitals], there is a mismatch between the centers of excess valence electron density and atomic positions in the electrides [38]. Due to the unbounded electronic states (called floating bands), electrides have two unique properties: (i) Electrides possess very low work functions, resulting in a wide variety of technological applications, including excellent electron emitters $[39,40]$ and superior catalysts $[41]$; and (ii) the floating bands usually are very close to the $E_{\mathrm{F}}$, which are very likely 
(a) spin- $1 / 2$ Weyl fermion $(\mathrm{C}=+1)$

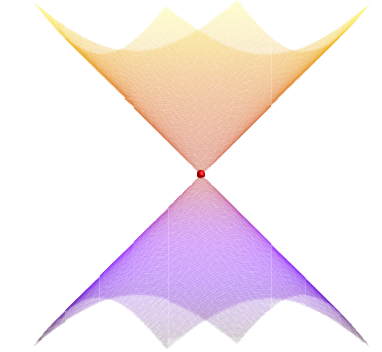

(c)

$$
\text { spin-1 excitation }
$$$$
(\mathrm{C}=+2)
$$

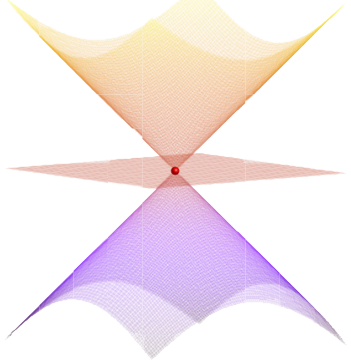

(b)

four-fold Dirac fermion $(\mathrm{C}=+1 \&-1)$

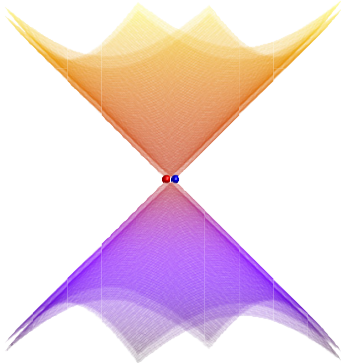

(d)

$$
\begin{aligned}
& \text { six-fold excitation } \\
& (\mathrm{C}=+2 \text { \& }-2)
\end{aligned}
$$

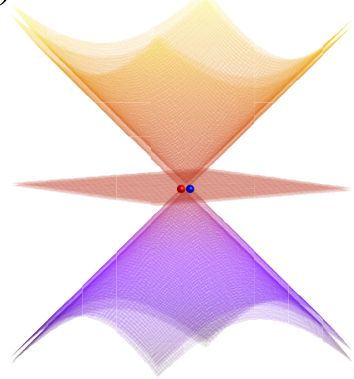

FIG. 1. Schematics of different types of fermions. (a) Spin-1/2 Weyl fermion with charge $C=+1$. (b) Fourfold Dirac fermion. (c) Spin-1 excitation with charge $C=+2$. (d) Sixfold excitation.

to interact with other energy bands, resulting in a band inversion and nontrivial band topology $[42,43]$. Because of the potential candidates of various topological states, electrides have received growing attention recently [44-49].

Here, we show that electride $\mathrm{Li}_{12} \mathrm{Mg}_{3} \mathrm{Si}_{4}$ exhibits a single linear dispersive sixfold excitation at the $H$ point near $E_{\mathrm{F}}$, which is formed by the unbounded states of the excess electrons centered at the vacancies, i.e., the $12 a$ Wyckoff sites (WKS). These floating bands correspond to the elementary band representation (eBR) of $A @ 12 a$, in the topological quantum chemistry (TQC) theory $[42,50]$. We also reveal a unique topological bulk-surface-edge correspondence for the excitation, leading to trivial surface "Fermi arcs" and topological hinge arcs. All energetically gapped $k_{z}$ slices belong to a 2D higher-order topological insulating (HOTI) phase. As a critical point of phase transitions, this state can be driven into various topological phases by breaking specific symmetries.

Crystal and electronic structures. $\mathrm{Li}_{12} \mathrm{Mg}_{3} \mathrm{Si}_{4}$ crystallizes in a body-centered-cubic structure with lattice constant $a=$ $10.688 \AA$ And SG $I \overline{4} 3 d$ (SG 220) [51], as shown in Fig. 2(a). Figure 2(d) shows the band structure of $\mathrm{Li}_{12} \mathrm{Mg}_{3} \mathrm{Si}_{4}$ without SOC along the high-symmetry $k$ points labeled in the first BZ of Fig. 2(b) [see details on the crystal structure and calculation methods in Secs. A and B of the Supplemental Material (SM) [52], respectively]. One can notice that the set of 24 VBs in the energy range -6 to $-0.6 \mathrm{eV}$ are well separated from other bands. In addition, there are two VBs right below $E_{\mathrm{F}}$, which are connecting to four conduction bands at the $H$ point. These six bands are colored in blue in Fig. 2(d), which are energetically isolated from higher-energy bands, as shown in Fig. 2(e). In the following, we will show that the six bluecolored bands do not belong to any atomic-orbital-induced (a)
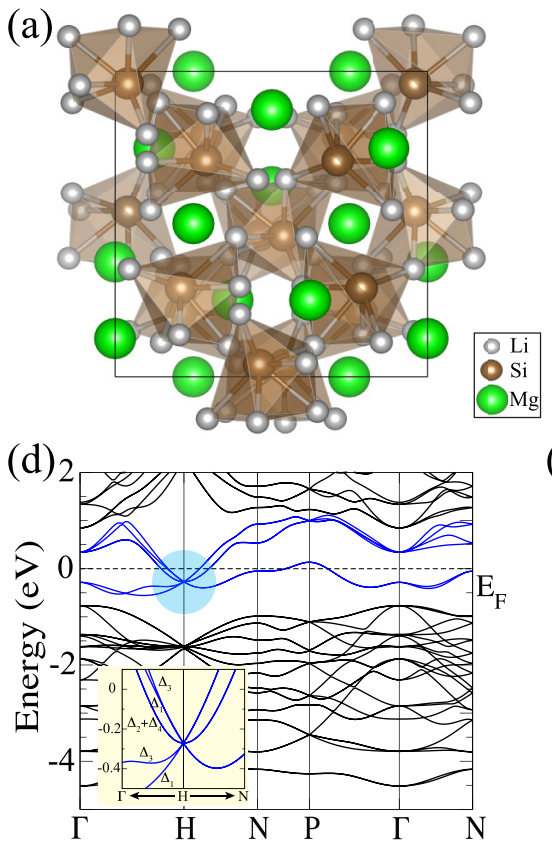

(b)

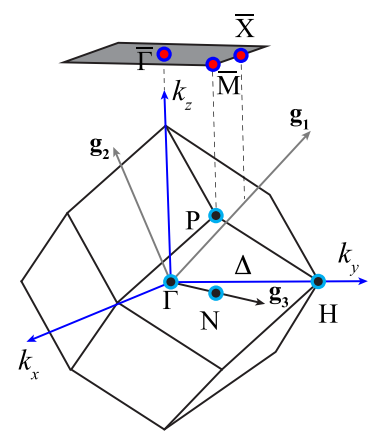

(e) 1.2

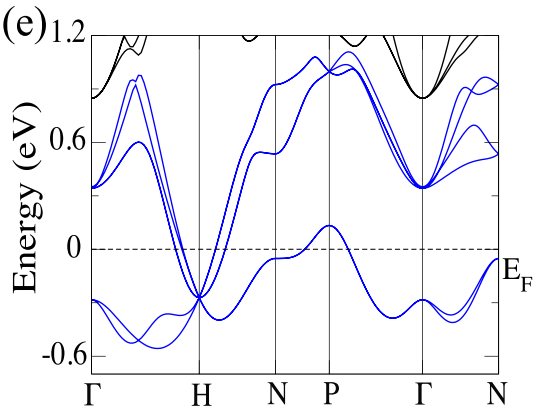

(c)

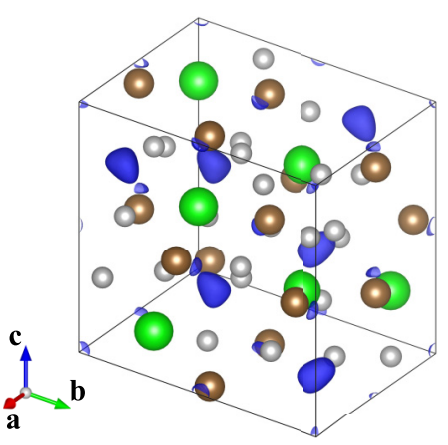

(f)

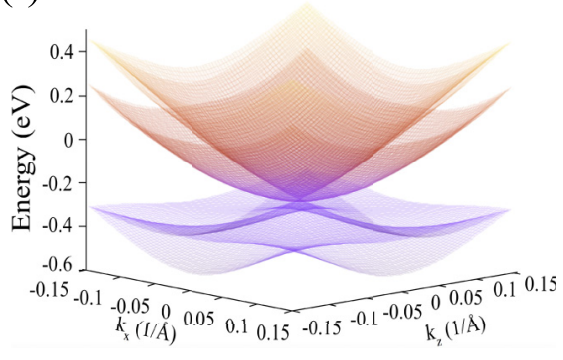

FIG. 2. Crystal and electronic structures of $\mathrm{Li}_{12} \mathrm{Mg}_{3} \mathrm{Si}_{4}$. (a) Top view of the crystal structure of $\mathrm{Li}_{12} \mathrm{Mg}_{3} \mathrm{Si}_{4}$. (b) Bulk BZ and (001) surface BZ with high-symmetry points indicated. (c) PED with an isosurface value of $0.0068 \mathrm{bohr}^{-3}$ for the six blue-colored bands in (d) or (e). The colored balls indicate the positions of the atoms, while the irregular surfaces in blue indicate the charge distribution around the $12 a$ sites. (d) and (e) The first-principles band structures of $\mathrm{Li}_{12} \mathrm{Mg}_{3} \mathrm{Si}_{4}$ without $\mathrm{SOC}$ in different energy ranges. The inset in (d) shows the zoom-in band structure around the sixfold excitation. (f) $2 \mathrm{D}$ band structure in the xoz plane around the sixfold excitation of the $H$ point. 
band representation (aBR), which can be generated by the code pos2aBR [37].

In the recently developed TQC theory, a complete list of eBRs has been constructed [released on the Bilbao Crystalline Server (BCS) [53]], serving as its basic building blocks, which link the irreducible representations (irreps) in momentum space and the orbital characters in real space directly (i.e., the BR of $\rho @ \delta$ is formed by the states of irrep $\rho$ centered at the WKS $\delta$ ). For topological materials, the irreps of their occupied bands cannot be decomposed into a sum of eBRs. On the other hand, for those trivial materials (a sum of eBRs), the decomposition of BRs can reveal orbital characters in real space, i.e., the average charge centers and site-symmetry characters, which was largely neglected before. In fact, the BR analysis provides a way to identify the origin of the bands around $E_{\mathrm{F}}$ from their symmetry eigenvalues (or irreps) alone. As such it opens up promising possibilities to predict electrides in the future [37] (see more details in Sec. C of the SM).

The irreps of the 30 electronic states at high-symmetry $k$ points shown in Table S2 are obtained by the program irvsp [54]. Comparing them with the BRs in TQC listed on BCS [53], we find that, as expected, the 24 lower bands belong to the aBRs of $\left(A_{1} \oplus^{1} E^{2} E\right) @ 16 c$, originating from Si $p$ orbitals. Interestingly, we also find that the six blue-colored bands belong to the BR of $A @ 12 a$, which is not an aBR because the $12 a$ WKS is empty. Thus, one can conjecture that $\mathrm{Li}_{12} \mathrm{Mg}_{3} \mathrm{Si}_{4}$ can be an electride with excess electrons serving as anions at the vacancies of the $12 a$ sites in terms of the representation theory. Furthermore, we calculate the partial electron density (PED) for these six bands. The results shown in Fig. 2(c) indicate the existence of a charge distribution around the $12 a$ sites [55], agreeing well with the analysis of BRs.

Then, we focus on the six bands of the $A @ 12 a$ BR near $E_{\mathrm{F}}$, especially the sixfold degenerate state at the $H$ point. The linear dispersive bands around the point resemble a sixfold excitation [highlighted in a shadowed circle in Fig. 2(d)]. Weyl nodes are coming in pairs with opposite chirality in Weyl semimetals. In 2D graphene, twofold Dirac fermions are also formed at two nonequivalent $K$ points. However, the state with a single linear dispersive spinless sixfold excitation in $\mathrm{Li}_{12} \mathrm{Mg}_{3} \mathrm{Si}_{4}$ is actually located at the phase boundary and protected by both nonsymmorphic crystalline symmetries and time-reversal symmetry $(\mathcal{T})$.

Symmetry analysis and sixfold excitations. First, along the $\Gamma-H$ line in Fig. 2(b) [i.e., the [100] $(\Delta)$ direction], the sixfold degenerate bands split into four singly degenerate bands and one doubly degenerate band, whose irreps can be labeled by the point group $C_{2 v}$, as shown in the inset of Fig. 2(d). Second, they split into three twofold degenerate bands along the $H-N$ line ([110] direction). It turns out that along the $H-N$ line, all bands are doubly degenerate due to the combined antiunitary symmetry satisfying $\left(\mathcal{T} \widetilde{M}_{1-10}\right)^{2}=-1$ (with $\widetilde{M}_{1-10} \equiv\left\{I C_{2,110} \mid \frac{1}{2},-\frac{1}{2}, \frac{1}{2}\right\}$; see the definitions for symmetry operators in Sec. A of the SM [52]).

Next, we investigate the symmetry protection of the sixfold excitation by studying the effective $\mathbf{k} \cdot \mathbf{p}$ Hamiltonian at the $H$ point, which is also shown with the Wilson-loop technique in Sec. F of the SM [52]. The nonsymmorphic little group of the $H$ point has three unitary operators $\left\{I C_{4 x}^{-1} \mid \frac{1}{2}, 0,0\right\}$, $\left\{I C_{2,110} \mid \frac{1}{2}, 0,0\right\},\left\{C_{3,1 \overline{1} \mid}^{-1} \mid 1, \frac{1}{2}, \frac{1}{2}\right\}$, and an antiunitary $\mathcal{T}$. The irreps of the six bands at $H$ are $H_{4} H_{5}$ in the convention of the BCS notation $[54,56]\left(H_{4}\right.$ and $H_{5}$ stick together due to $\mathcal{T}$ ). Under the basis of $\mathrm{H}_{4} \mathrm{H}_{5}$ irreps, the matrix representations of the four symmetries are given in Sec. D of the SM [52], explicitly.

After the consideration of all symmetry restrictions, the low-energy effective model can be up to the first order of $\delta \mathbf{k}$ (offset from the $H$ point) written as

$$
\mathcal{H}(\delta \mathbf{k})=\left(\begin{array}{cc}
p H_{3}\left(0, \delta \mathbf{k}^{\prime}\right) & -i q^{*} H_{3}\left(\pi / 2, \delta \mathbf{k}^{\prime}\right) \\
i q H_{3}\left(\pi / 2, \delta \mathbf{k}^{\prime}\right) & -p H_{3}\left(0, \delta \mathbf{k}^{\prime}\right)
\end{array}\right),
$$

where $\delta \mathbf{k}^{\prime} \equiv\left(\delta k_{x},-\delta k_{y},-\delta k_{z}\right), p$ has to be a real parameter, $q$ is an arbitrary parameter. and the full expression of $H_{3}$ is shown in Sec. E of the SM [52]. When $\phi=\pi / 2, H_{3}$ takes the form of $H_{3}(\pi / 2, \delta \mathbf{k})=\delta \mathbf{k} \cdot \mathbf{S}$, where the matrices $\mathbf{S}$ are the generators of the rotation group $\mathrm{SO}(3)$ in the spin-1 representation. In the limit of $|q| \gg|p|(|p| \gg|q|)$, this sixfold degenerate state consists of two threefold spin-1 excitations [two threefold excitations of $H_{3}\left(0, \delta \mathbf{k}^{\prime}\right)$ ], which are related by $\mathcal{T}$. By matching the first-principles band structure of $\mathrm{Li}_{12} \mathrm{Mg}_{3} \mathrm{Si}_{4}$ with that obtained from the model $\alpha \delta \mathbf{k}^{2}+\mathcal{H}(\delta \mathbf{k})$, the parameters $\alpha, p$, and $q$ are fitted to be $12 \mathrm{eV} \AA^{2}, 0.2 \mathrm{eV} \AA$, and $2.2 \mathrm{eV} \AA$, respectively. It is worth noting that the splittings of the sixfold degenerate state are only related to the linear terms $[\mathcal{H}(\delta \mathbf{k})]$, and the purpose for considering the quadratic term $\left(\alpha \delta \mathbf{k}^{2}\right)$ is to fit the bands well. Thus, the spinless sixfold degenerate state in $\mathrm{Li}_{12} \mathrm{Mg}_{3} \mathrm{Si}_{4}$ is in the limit of two spin-1 excitations with opposite chirality (i.e., the sixfold excitation), leading to the net topological charge of the two lowest bands being zero. This state is different from the spinful sixfold excitations in SGs 205, 206, and 230 with double degeneracy in all directions, and those in SGs 198, 212, and 213 consisting of two spin-1 excitations with the same chirality [21].

Tight-binding model, surface states, and hinge arc states. The sixfold excitation can be well reproduced by a tightbinding (TB) model with an $s$ orbital at the $12 a$ WKS. It reads as follows,

$$
H_{\mathrm{TВ}}(\mathbf{k})=\epsilon_{0}+\sum_{|\mathbf{r}|=d_{j=1,2,3}} t_{j} e^{i \mathbf{k} \cdot \mathbf{r}},
$$

where $\epsilon_{0}$ is the on-site energy of the $s$ orbital, $t_{1,2,3}$ are the nearest-, next-nearest-, and next-next-nearest-neighbor hopping parameters with the distances $d_{1,2,3}$, respectively. By fitting with the first-principles band structure in the vicinity of the $H$ point, the parameters of the TB band structure plotted in Fig. 3(a) are obtained, which are given in Sec. G of the SM (SM G). A single sixfold excitation emerges at the $H$ point, whose dispersions in all directions are consistent with first-principles calculations. By using the Green's function on a semi-infinite structure, the (001) surface dispersions are obtained in Fig. 3(b), where four surface states are terminated at the projection of the sixfold excitation (i.e., $\bar{\Gamma}$ ). As the sixfold excitation does not host net Berry curvature, there is no guarantee of topologically protected surface states in the strictest sense. However, the trivial surface Fermi arcs (i.e., their starting point and ending point coincide) associated with the sixfold excitation are usually expected in the related materials (unless they are finely tuned), similar to the case in a Dirac semimetal (DSM) (see more details in SM G). 


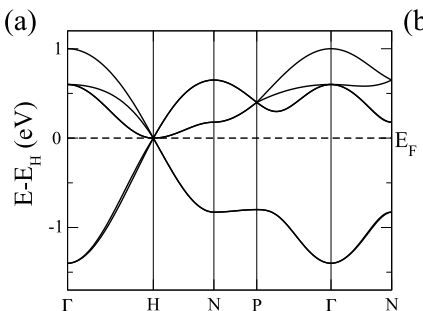

(c)
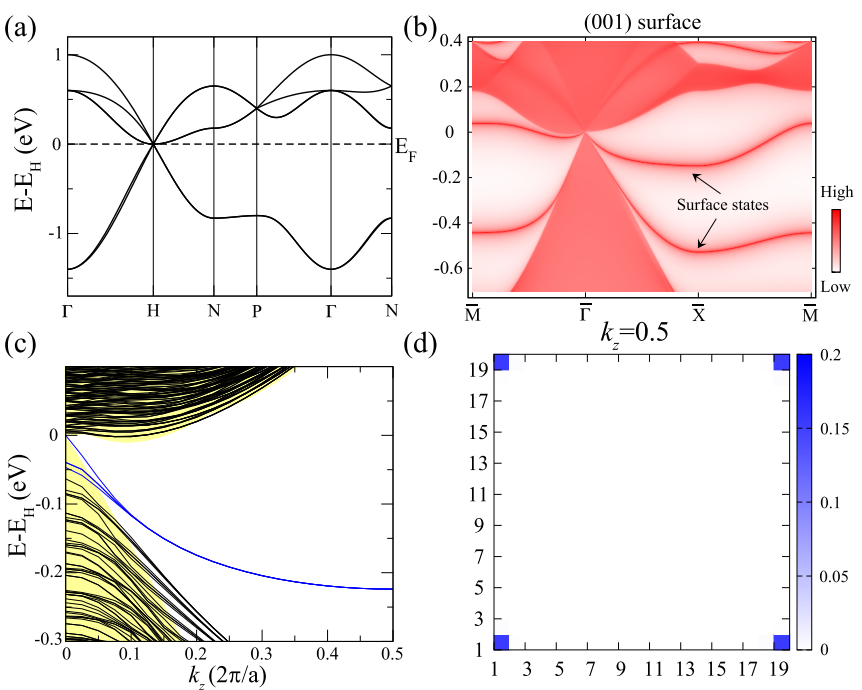

(d)

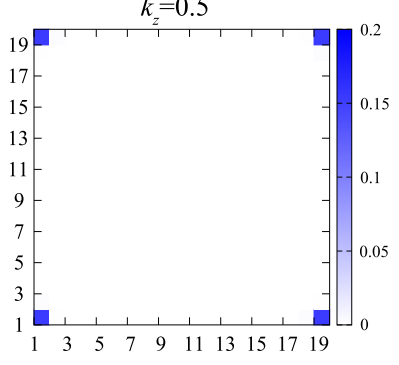

FIG. 3. The bulk and boundary states of the TB Hamiltonian. (a) The bulk bands, (b) (001) surface dispersions, and (c) [001] hinge dispersions. The yellow shadow denotes the projections of the bulk states. (d) The real-space distributions of the four hinge states for $k_{z}=\frac{\pi}{a}$ on the structure of a 1D $20 \times 20$ supercell.

Although the surface states of $\mathrm{Li}_{12} \mathrm{Mg}_{3} \mathrm{Si}_{4}$ are not topologically protected, there are topological hinge arc states on its [001] hinges, as analogous to the hinge arcs in DSMs. In DSMs, all energetically gapped $k_{z}$ slices are classified into two different 2D phases (one is trivial, while the other is a 2D topological HOTI phase), which are separated by a gapless Dirac point [26]. Interestingly, in semimetals with a single spinless sixfold excitation, all gapped $k_{z}$ slices belong to a $2 \mathrm{D}$ HOTI phase, except for the gapless plane with sixfold excitation. The nontrivial topology of a 2D HOTI phase is closely related to a filling anomaly [57]: a mismatch between the number of electrons required to simultaneously satisfy charge neutrality and the crystal symmetry (i.e., $\mathcal{T} \widetilde{S}_{4 z}$ ). By computing one-dimensional (1D) Wilson loops along the $\mathbf{b}_{1}^{*}\left(\equiv \mathbf{g}_{3}\right)$ and $\mathbf{b}_{2}^{*}\left(\equiv \mathbf{g}_{1}-\mathbf{g}_{2}\right)$ directions, our results show that the two charge centers are quantized to $\left[0.25 \mathbf{b}_{1}, 0.25 \mathbf{b}_{2}\right]$ and $\left[0.75 \mathbf{b}_{1}, 0.75 \mathbf{b}_{2}\right]$ with $\mathbf{b}_{1} \equiv\{a / 2, a / 2\}$ and $\mathbf{b}_{2} \equiv\{-a / 2, a / 2\}$, which correspond to $\{0,0.25 a\}$ and $\{0,0.75 a\}$, respectively, in Cartesian coordinates in the projected $x y$ plane. The quantizations are protected by the $\mathcal{T} \widetilde{S}_{4 z}$ symmetry (with $\widetilde{S}_{4 z} \equiv\left\{I C_{4 z}^{-1} \mid 00 \frac{1}{2}\right\}$ ) for each gapped $k_{z}$ slice. Because the two obtained Wannier charge centers are two distinct $\mathcal{T} \widetilde{S}_{4 z}$-invariant positions, it is impossible to have any choice of Wannier center assignment that preserves charge neutrality and $\widetilde{S}_{4 z}$ symmetry simultaneously [57].

Unlike the chiral/helical hinge modes connecting the conduction bands and VBs in a 3D HOTI phase, the in-gap hinge arcs of $\mathrm{Li}_{12} \mathrm{Mg}_{3} \mathrm{Si}_{4}$ are connecting the projection of the sixfold excitation between adjacent projected 1D BZs. In Fig. 3(c), we plot the [001] hinge dispersions of an 1D $\widetilde{S}_{4 z}$-invariant $20 \times 20$ supercell with open-boundary conditions in the $\mathbf{b}_{1}$ and $\mathbf{b}_{2}$ directions. The in-gap bands colored in blue are four degenerate hinge states, which are well localized on four hinges, respectively. The real-space distributions of the four hinge states for $k_{z}=\frac{\pi}{a}$ are shown in Fig. 3(d) (see Sec. H of the SM [52]). By simply counting the electronic bands, three electrons occupy the fourfold-degenerate hinge states in a nonzero $k_{z}$ plane $\left(k_{z} \neq 0\right)$, resulting in a fractional corner charge $Q_{\text {corner }}=\frac{3|e|}{4}$ [58-61]. Therefore, the [001]-hinge arc states, well separated from the bulk and surface projections, confirm nontrivial bulk topology in $\mathrm{Li}_{12} \mathrm{Mg}_{3} \mathrm{Si}_{4}$.

Phase transitions in $\mathrm{Li}_{12} \mathrm{Mg}_{3} \mathrm{Si}_{4}$. The state with a single sixfold excitation can be driven into various topological states after consideration of the strain effect, which can be simulated through multiplying the hopping parameters in the Hamiltonian by a factor of $\left|\mathbf{r}^{\prime} / \mathbf{r}\right|$ with $\mathbf{r}\left(\mathbf{r}^{\prime}\right)$ the distances of the hoppings without (with) strains. Here, the compressive strains along the [001] and [111] directions are studied, and the band dispersions near $H$ are shown in Figs. 4(a)-4(c). When strain is applied in the [001] direction, the sixfold excitation splits into four Weyl points in the $k_{z}=0$ plane, whose projections on the (001) surface are the ends of two long visible Fermi-arc states extending through the BZ in spite of the small splitting of Weyl points [Figs. S4(b) and S4(c) and Figs. S5(b) and $\mathrm{S} 5(\mathrm{c})$ in SM G [52]]. On the other hand, once strain is applied in the [111] direction, a phase transition from a single sixfold excitation to two sets of nodal lines is observed. Each set of nodal lines consists of one line segment in the [111] direction and three $C_{3,111}$-related arc segments [Figs. S6(b) and S6(c); see symmetry protections in SM G].

In summary, the electride $\mathrm{Li}_{12} \mathrm{Mg}_{3} \mathrm{Si}_{4}$ hosting a single linear dispersive sixfold excitation near $E_{\mathrm{F}}$ can be well understood by the analysis of BRs in TQC. The sixfold excitation
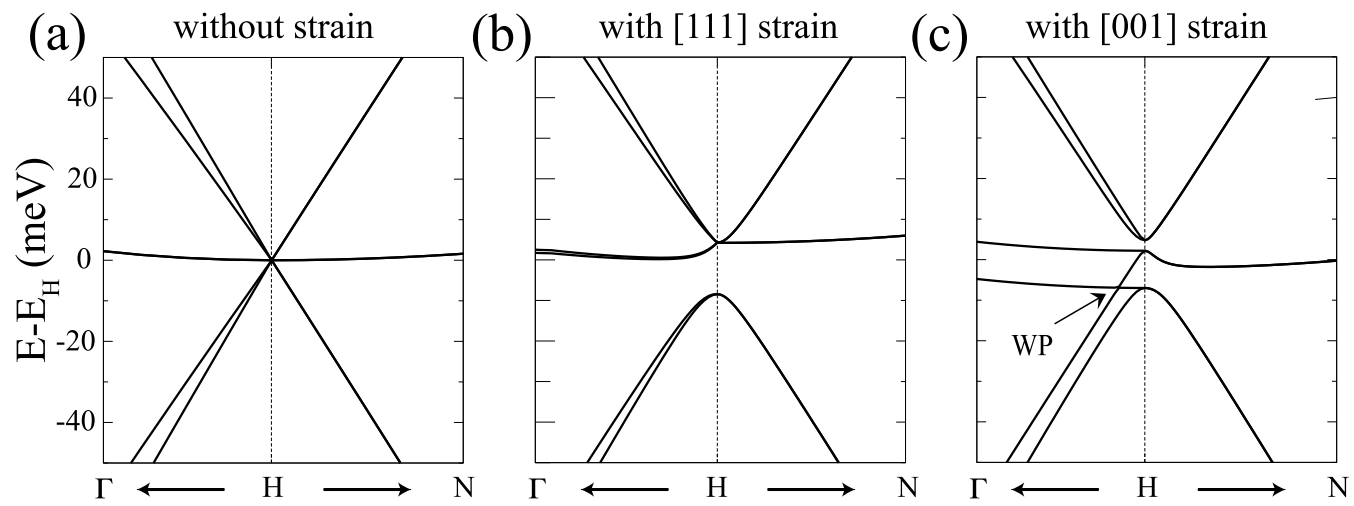

FIG. 4. The bulk band structures (a) without strain, (b) with [111] strain, and (c) with [001] strain. 
is formed by the floating states of the unbounded electrons in $\mathrm{Li}_{12} \mathrm{Mg}_{3} \mathrm{Si}_{4}$, labeled by the BR of $A @ 12 a$ with an $s$-like pseudo-orbital (i.e., $A$ irrep) at the $12 a$ WKS. Given the unique topological bulk-surface-edge correspondence for the excitation, trivial Fermi-arc surface states and topological hinge arc states are obtained. Moreover, the fact that it is very clean around $E_{\mathrm{F}}$ in these materials offers a good chance for the study of transport and photogalvanic experiments. When the strain effect is considered, the sixfold excitation can be driven into Weyl points or nodal lines, etc. Due to the negligible strength of SOC, a 12-fold degenerate excitation (the highest degeneracy in electronic systems) can be expected in $\mathrm{Li}_{12} \mathrm{Mg}_{3} \mathrm{Si}_{4}$ after consideration of the spin degrees of freedom (see Sec. I of the SM [52]). Moreover, there is a rich family of $A_{12} A_{3}^{\prime} B_{4}$ compounds, including $\mathrm{Li}_{12} \mathrm{Mg}_{3} \mathrm{Si}_{4}, \mathrm{Li}_{12} \mathrm{Al}_{3} \mathrm{Si}_{4}$, and $\mathrm{Li}_{15} \mathrm{Ge}_{4}$, etc. (see Sec. I of the SM [52]), making the chemical potential of $\mathrm{Li}_{12} \mathrm{Mg}_{3} \mathrm{Si}_{4}$ experimentally tunable. These electrides also serve as different platforms for the study of the topological semimetallic states and phase transitions in future experiments.

Acknowledgments. This work was supported by the National Natural Science Foundation of China (Grant No. 11974395), the Strategic Priority Research Program of Chinese Academy of Sciences (Grant No. XDB33000000), and the Center for Materials Genome. B.A.B. was supported by the Department of Energy Grant No. DE-SC0016239, and additionally supported by the National Science Foundation EAGER Grant No. DMR 1643312, Simons Investigator Grant No. 404513, ONR Grant No. N00014-14-1-0330, the Packard Foundation, the Schmidt Fund for Innovative Research, and a Guggenheim Fellowship from the John Simon Guggenheim Memorial Foundation.
[1] P. B. Pal, Dirac, Majorana, and Weyl fermions, Am. J. Phys. 79, 485 (2011).

[2] Z. Wang, Y. Sun, X.-Q. Chen, C. Franchini, G. Xu, H. Weng, $X$. Dai, and Z. Fang, Three-dimensional Dirac semimetal and quantum transport in $\mathrm{Cd}_{3} \mathrm{As}_{2}$, Phys. Rev. B 85, 195320 (2012).

[3] Z. Wang, H. Weng, Q. Wu, X. Dai, and Z. Fang, Threedimensional Dirac semimetal and quantum transport in $\mathrm{Cd}_{3} \mathrm{As}_{2}$, Phys. Rev. B 88, 125427 (2013).

[4] S. M. Young, S. Zaheer, J. C. Y. Teo, C. L. Kane, E. J. Mele, and A. M. Rappe, Dirac Semimetal in Three Dimensions, Phys. Rev. Lett. 108, 140405 (2012).

[5] P. Tang, Q. Zhou, G. Xu, and S.-C. Zhang, Dirac fermions in an antiferromagnetic semimetal, Nat. Phys. 12, 1100 (2016).

[6] G. Hua, S. Nie, Z. Song, R. Yu, G. Xu, and K. Yao, Dirac semimetal in type-IV magnetic space groups, Phys. Rev. B 98, 201116(R) (2018).

[7] X. Wan, A. M. Turner, A. Vishwanath, and S. Y. Savrasov, Topological semimetal and Fermi-arc surface states in the electronic structure of pyrochlore iridates, Phys. Rev. B 83, 205101 (2011).

[8] G. Xu, H. Weng, Z. Wang, X. Dai, and Z. Fang, Chern Semimetal and the Quantized Anomalous Hall Effect in $\mathrm{HgCr}_{2} \mathrm{Se}_{4}$, Phys. Rev. Lett. 107, 186806 (2011).

[9] H. Weng, C. Fang, Z. Fang, B. A. Bernevig, and X. Dai, Weyl Semimetal Phase in Noncentrosymmetric TransitionMetal Monophosphides, Phys. Rev. X 5, 011029 (2015).

[10] S.-M. Huang, S.-Y. Xu, I. Belopolski, C.-C. Lee, G. Chang, B. Wang, N. Alidoust, G. Bian, M. Neupane, C. Zhang et al., A Weyl Fermion semimetal with surface Fermi arcs in the transition metal monopnictide TaAs class, Nat. Commun. 6, 7373 (2015).

[11] Z. Wang, M. G. Vergniory, S. Kushwaha, M. Hirschberger, E. V. Chulkov, A. Ernst, N. P. Ong, R. J. Cava, and B. A. Bernevig, Time-Reversal-Breaking Weyl Fermions in Magnetic Heusler Alloys, Phys. Rev. Lett. 117, 236401 (2016).

[12] S. Nie, G. Xu, F. B. Prinz, and S.-c. Zhang, Topological semimetal in honeycomb lattice LnSI, Proc. Natl. Acad. Sci. USA 114, 10596 (2017).

[13] Q. Wang, Y. Xu, R. Lou, Z. Liu, M. Li, Y. Huang, D. Shen, H. Weng, S. Wang, and H. Lei, Large intrinsic anomalous Hall effect in half-metallic ferromagnet $\mathrm{Co}_{3} \mathrm{Sn}_{2} \mathrm{~S}_{2}$ with magnetic Weyl fermions, Nat. Commun. 9, 1 (2018).

[14] S. Nie, Y. Sun, F. B. Prinz, Z. Wang, H. Weng, Z. Fang, and X. Dai, Magnetic Semimetals and Quantized Anomalous Hall Effect in $\mathrm{EuB}_{6}$, Phys. Rev. Lett. 124, 076403 (2020).

[15] A. A. Soluyanov, D. Gresch, Z. Wang, Q. Wu, M. Troyer, X. Dai, and B. A. Bernevig, Type-II Weyl semimetals, Nature (London) 527, 495 (2015).

[16] S. Nadj-Perge, I. K. Drozdov, J. Li, H. Chen, S. Jeon, J. Seo, A. H. MacDonald, B. A. Bernevig, and A. Yazdani, Observation of Majorana fermions in ferromagnetic atomic chains on a superconductor, Science 346, 602 (2014).

[17] Q. L. He, L. Pan, A. L. Stern, E. C. Burks, X. Che, G. Yin, J. Wang, B. Lian, Q. Zhou, E. S. Choi et al., Chiral Majorana fermion modes in a quantum anomalous Hall insulator-superconductor structure, Science 357, 294 (2017).

[18] P. Zhang, K. Yaji, T. Hashimoto, Y. Ota, T. Kondo, K. Okazaki, Z. Wang, J. Wen, G. Gu, H. Ding et al., Observation of topological superconductivity on the surface of an iron-based superconductor, Science 360, 182 (2018).

[19] D. Wang, L. Kong, P. Fan, H. Chen, S. Zhu, W. Liu, L. Cao, Y. Sun, S. Du, J. Schneeloch et al., Evidence for Majorana bound states in an iron-based superconductor, Science 362, 333 (2018).

[20] J. Ma, C. Yi, B. Lv, Z. Wang, S. Nie, L. Wang, L. Kong, Y. Huang, P. Richard, P. Zhang et al., Experimental evidence of hourglass fermion in the candidate nonsymmorphic topological insulator KHgSb, Sci. Adv. 3, e1602415 (2017).

[21] B. Bradlyn, J. Cano, Z. Wang, M. G. Vergniory, C. Felser, R. J. Cava, and B. A. Bernevig, Beyond Dirac and Weyl fermions: Unconventional quasiparticles in conventional crystals, Science 353, aaf5037 (2016).

[22] P. Tang, Q. Zhou, and S.-C. Zhang, Multiple Types of Topological Fermions in Transition Metal Silicides, Phys. Rev. Lett. 119, 206402 (2017).

[23] G. Chang, S.-Y. Xu, B. J. Wieder, D. S. Sanchez, S.-M. Huang, I. Belopolski, T.-R. Chang, S. Zhang, A. Bansil, H. Lin et al., Unconventional Chiral Fermions and Large Topological Fermi Arcs in RhSi, Phys. Rev. Lett. 119, 206401 (2017). 
[24] T. Zhang, Z. Song, A. Alexandradinata, H. Weng, C. Fang, L. Lu, and Z. Fang, Double-Weyl Phonons in Transition-Metal Monosilicides, Phys. Rev. Lett. 120, 016401 (2018).

[25] W. Rarita and J. Schwinger, On a Theory of Particles with HalfIntegral Spin, Phys. Rev. 60, 61 (1941).

[26] B. J. Wieder, Z. Wang, J. Cano, X. Dai, L. M. Schoop, B. Bradlyn, and B. A. Bernevig, Strong and fragile topological Dirac semimetals with higher-order Fermi arcs, Nat. Commun. 11, 627 (2020).

[27] Z. Rao, H. Li, T. Zhang, S. Tian, C. Li, B. Fu, C. Tang, L. Wang, Z. Li, W. Fan et al., Observation of unconventional chiral fermions with long Fermi arcs in CoSi, Nature (London) 567, 496 (2019)

[28] D. Takane, Z. Wang, S. Souma, K. Nakayama, T. Nakamura, H. Oinuma, Y. Nakata, H. Iwasawa, C. Cacho, T. Kim et al., Observation of Chiral Fermions with a Large Topological Charge and Associated Fermi-Arc Surface States in CoSi, Phys. Rev. Lett. 122, 076402 (2019).

[29] N. Kumar, M. Yao, J. Nayak et al., Signatures of sixfold degenerate exotic fermions in a superconducting metal $\mathrm{PdSb}_{2}, \mathrm{Adv}$. Mater. 32, 1906046 (2020).

[30] Z. P. Sun, C. Q. Hua, X. L. Liu, Z. T. Liu, M. Ye, S. Qiao, Z. H. Liu, J. S. Liu, Y. F. Guo, Y. H. Lu et al., Direct observation of sixfold exotic fermions in the pyrite-structured topological semimetal $\mathrm{PdSb}_{2}$, Phys. Rev. B 101, 155114 (2020).

[31] X. Yáng, T. A. Cochran, R. Chapai, D. Tristant, J.-X. Yin, I. Belopolski, Z. Chéng, D. Multer, S. S. Zhang, N. Shumiya, M. Litskevich, Y. Jiang, G. Chang, Q. Zhang, I. Vekhter, W. A. Shelton, R. Jin, S.-Y. Xu, and M. Z. Hasan, Observation of sixfold degenerate fermions in $\mathrm{PdSb}_{2}$, Phys. Rev. B 101, 201105(R) (2020).

[32] R. Chapai, Y. Jia, W. A. Shelton, R. Nepal, M. Saghayezhian, J. F. DiTusa, E. W. Plummer, C. Jin, and R. Jin, Fermions and bosons in nonsymmorphic $\mathrm{PdSb}_{2}$ with sixfold degeneracy, Phys. Rev. B 99, 161110(R) (2019).

[33] S. B. Dawes, D. L. Ward, R. H. Huang, and J. L. Dye, First electride crystal structure, J. Am. Chem. Soc. 108, 3534 (1986).

[34] J. L. Dye and M. G. DeBacker, Physical and chemical properties of alkalides and electrides, Annu. Rev. Phys. Chem. 38, 271 (1987).

[35] S. Matsuishi, Y. Toda, M. Miyakawa, K. Hayashi, T. Kamiya, M. Hirano, I. Tanaka, and H. Hosono, High-density electron anions in a nanoporous single crystal: $\left[\mathrm{Ca}_{24} \mathrm{Al}_{28} \mathrm{O}_{64}\right]^{4+}\left(4 e^{-}\right)$, Science 301, 626 (2003).

[36] K. Lee, S. W. Kim, Y. Toda, S. Matsuishi, and H. Hosono, Dicalcium nitride as a two-dimensional electride with an anionic electron layer, Nature (London) 494, 336 (2013).

[37] S. Nie, Y. Qian, J. Gao, Z. Fang, H. Weng, and Z. Wang, The application of topological quantum chemistry in electrides, arXiv:2012.02203.

[38] D. J. Singh, H. Krakauer, C. Haas, and W. E. Pickett, Theoretical determination that electrons act as anions in the electride $\mathrm{Cs}^{+}(15 \text {-crown-5) })_{2} \mathrm{e}^{-}$, Nature (London) 365, 39 (1993).

[39] R. C. Phillips, W. P. Pratt, and J. L. Dye, Thermionic emission from cold electride films, Chem. Mater. 12, 3642 (2000).

[40] R. H. Huang and J. L. Dye, Low temperature $\left(-80{ }^{\circ} \mathrm{C}\right)$ thermionic electron emission from alkalides and electrides, Chem. Phys. Lett. 166, 133 (1990).
[41] M. Kitano, Y. Inoue, Y. Yamazaki, F. Hayashi, S. Kanbara, S. Matsuishi, T. Yokoyama, S.-W. Kim, M. Hara, and H. Hosono, Ammonia synthesis using a stable electride as an electron donor and reversible hydrogen store, Nat. Chem. 4, 934 (2012).

[42] B. Bradlyn, L. Elcoro, J. Cano, M. Vergniory, Z. Wang, C. Felser, M. Aroyo, and B. A. Bernevig, Topological quantum chemistry, Nature (London) 547, 298 (2017).

[43] H. C. Po, A. Vishwanath, and H. Watanabe, Symmetry-based indicators of band topology in the 230 space groups, Nat. Commun. 8, 1 (2017).

[44] H. Huang, K.-H. Jin, S. Zhang, and F. Liu, Symmetry-based indicators of band topology in the 230 space groups, Nano Lett. 18, 1972 (2018).

[45] M. Hirayama, S. Matsuishi, H. Hosono, and S. Murakami, Electrides as a New Platform of Topological Materials, Phys. Rev. X 8, 031067 (2018).

[46] S.-C. Zhu, L. Wang, J.-Y. Qu, J.-J. Wang, T. Frolov, X.-Q. Chen, and Q. Zhu, Computational design of flexible electrides with nontrivial band topology, Phys. Rev. Materials 3, 024205 (2019).

[47] X. Zhang, B. Fu, L. Jin, X. Dai, G. Liu, and Y. Yao, Topological nodal line electrides: Realization of an ideal nodal line state nearly immune from spin-orbit coupling, J. Phys. Chem. C 123, 25871 (2019)

[48] S. Nie, H. Weng, and F. B. Prinz, Topological nodal-line semimetals in ferromagnetic rare-earth-metal monohalides, Phys. Rev. B 99, 035125 (2019).

[49] J. Wang, X. Sui, S. Gao, W. Duan, F. Liu, and B. Huang, Anomalous Dirac Plasmons in 1D Topological Electrides, Phys. Rev. Lett. 123, 206402 (2019).

[50] B. Bradlyn, L. Elcoro, M. G. Vergniory, J. Cano, Z. Wang, C. Felser, M. I. Aroyo, and B. A. Bernevig, Band connectivity for topological quantum chemistry: Band structures as a graph theory problem, Phys. Rev. B 97, 035138 (2018).

[51] D. Kevorkov, R. Schmid-Fetzer, and F. Zhang, Phase equilibria and thermodynamics of the Mg-Si-Li system and remodeling of the Mg-Si system, J. Phase Equilib. Diffus. 25, 140 (2004).

[52] See Supplemental Material at http://link.aps.org/supplemental/ 10.1103/PhysRevResearch.3.L012028 for the details on the vectors of SG 220 and structure information of $\mathrm{Li}_{12} \mathrm{Mg}_{3} \mathrm{Si}_{4}$, calculation method, introduction to TQC and its application in electrides, representation matrices of the generators at $H$, band dispersions of the low-energy effective $\mathbf{k} \cdot \mathbf{p}$ model, Wilsonloop technique, the TB Hamiltonian, surface states and strain effect, hinge states, and band structure of $\mathrm{Li}_{12} \mathrm{Mg}_{3} \mathrm{Si}_{4}$ with SOC and $A_{12} A_{3}^{\prime} B_{4}$ without SOC.

[53] Bilbao crystallographic server, https://www.cryst.ehu.es/cgibin/cryst/programs/bandrep.pl.

[54] J. Gao, Q. Wu, C. Persson, and Z. Wang, Irvsp: To obtain irreducible representations of electronic states in the VASP, Comput. Phys. Commun. 261, 107760 (2021).

[55] J. Wang, Q. Zhu, Z. Wang, and H. Hosono, Ternary inorganic electrides with mixed bonding, Phys. Rev. B 99, 064104 (2019).

[56] H. T. Stokes, B. J. Campbell, and R. Cordes, Tabulation of irreducible representations of the crystallographic space groups and their superspace extensions, Acta Crystallogr., Sect. A: Found. Crystallogr. 69, 388 (2013).

[57] W. A. Benalcazar, T. Li, and T. L. Hughes, Quantization of fractional corner charge in $C_{n}$-symmetric higher-order topological crystalline insulators, Phys. Rev. B 99, 245151 (2019). 
[58] C. W. Peterson, W. A. Benalcazar, T. L. Hughes, and G. Bahl, A quantized microwave quadrupole insulator with topologically protected corner states, Nature (London) 555, 346 (2018).

[59] S. Franca, J. van den Brink, and I. C. Fulga, An anomalous higher-order topological insulator, Phys. Rev. B 98, 201114(R) (2018).
[60] H. Xue, Y. Yang, F. Gao, Y. Chong, and B. Zhang, Acoustic higher-order topological insulator on a kagome lattice, Nat. Mater. 18, 108 (2019).

[61] F. Schindler, M. Brzezińska, W. A. Benalcazar, M. Iraola, A. Bouhon, S. S. Tsirkin, M. G. Vergniory, and T. Neupert, Fractional corner charges in spin-orbit coupled crystals, Phys. Rev. Research 1, 033074 (2019). 